\title{
The sarcomeric protein nebulin: another multifunctional giant in charge of muscle strength optimization
}

\author{
Coen A. C. Ottenheijm ${ }^{1,2}$, Henk Granzier ${ }^{2}$ and Siegfried Labeit ${ }^{3}$ \\ ${ }^{1}$ Department of Physiology, Institute for Cardiovascular Research, VU University Medical Center, Amsterdam, Netherlands \\ ${ }^{2}$ Department of Physiology, University of Arizona, Tucson, AZ, USA \\ ${ }^{3}$ Department of Integrative Pathophysiology, Universitätsmedizin Mannheim, University of Heidelberg, Mannheim, Germany
}

\section{Edited by:}

Christina Karatzaferi, University of

Thessaly, Greece

\section{Reviewed by:}

Corrado Poggesi, University of

Florence, Italy

Velia M. Fowler, The Scripps Research

Institute, USA

*Correspondence:

Coen A. C. Ottenheijm, Department of Physiology, VU University Medical

Center, 1081 BT Amsterdam,

Netherlands.

e-mail: c.ottenheijm@vumc.nl
The sliding filament model of the sarcomere was developed more than half a century ago. This model, consisting only of thin and thick filaments, has been successful in explaining many, but not all, features of skeletal muscle. Work during the 1980s revealed the existence of two additional filaments: the giant filamentous proteins titin and nebulin. Whereas the role of titin rapidly progressed, nebulin's role in muscle structure and function remained long nebulous. An important feature of muscle structure and function that has remained relatively obscure concerns the mechanisms that are involved in regulating thin filament length. Filament length is an important aspect of muscle function as force production is proportional to the amount of overlap between thick and thin filaments. Recent advances, due in part to the generation of nebulin $\mathrm{KO}$ models, reveal that nebulin plays an important role in the regulation of thin filament length, most likely by stabilizing F-actin assemblies. Another structural feature of skeletal muscle that has been incompletely understood concerns the mechanisms involved in maintaining Z-disk structure and the regular lateral alignment of adjacent sarcomeres during contraction. Recent studies indicate that nebulin is part of a protein complex that mechanically links adjacent myofibrils. In addition to these structural roles in support of myofibrillar force generation, nebulin has been also shown to regulate directly muscle contraction at the level of individual crossbridges: cycling kinetics and the calcium sensitivity of force producing crossbridges is enhanced in the presence of nebulin. Thus, these recent data all point to nebulin being important for muscle force optimization. Consequently, muscle weakness as the lead symptom develops in the case of patients with nemaline myopathy that have mutations in the nebulin gene. Here, we discuss these important novel insights into the role of nebulin in skeletal muscle function.

\section{NEBULIN IS INVOLVED IN SPECIFYING THIN FILAMENT LENGTH}

Skeletal muscle produces efficient contractile force because they contain thin and thick filaments of well-defined lengths that are organized into regular, symmetric arrays that interdigitate. Filament length is an important aspect of muscle function because a muscle generates force in proportion to thin and thick filament overlap. Whereas thick filament length is considered a constant $1.6 \mu \mathrm{m}$, thin filament lengths are fine-tuned at $\sim 1.0-1.3 \mu \mathrm{m}$, depending on species and muscle type (Littlefield and Fowler, 2008) to overlap with thick filaments and to meet the muscle's physiological demands (Granzier et al., 1991; Burkholder et al., 1994; Littlefield and Fowler, 2008).

Changes in thin filament length affect thin-thick filament overlap and impact a muscle's force generating capacity at a given sarcomere length: thus, thin filament length is a key aspect of muscle function. Since length is not an intrinsic property of actin filaments (actin monomers assemble in vitro to highly variable polymer lengths (Pollard and Borisy, 2003), thin filament length is likely to be specified in vivo by an actin-binding protein; for this, nebulin has been considered for a long time a prime candidate (Wang and Wright, 1988; Labeit et al., 1991; Labeit and Kolmerer, 1995), but critical evidence has been lacking up until recently.

Nebulin is a giant protein $(\mathrm{Mw} 700-800 \mathrm{kDa})$ expressed in skeletal muscle, and makes up 2-3\% of the myofibrillar protein mass. Immuno-electron microscopy revealed that a single nebulin molecule spans the thin filament with its $\mathrm{C}$-terminus anchored at the Z-disk and its $\mathrm{N}$-terminal region directed toward the thin filament pointed-end (for a schematic representation, see Figure 1; Wang and Wright, 1988). The first evidence for nebulin's proposed role in specifying thin filament length came from the analysis of nebulin's cDNA sequence. This revealed that the bulk of the molecule is comprised of modules with the centrally located modules, M9 to M162, each thought to represent individual actin-binding motifs, and organized into seven-module super-repeats that match the repeat of the actin filament (Figure 1). This precise arrangement is thought to allow each nebulin module to interact with a single monomer of the actin filament (Labeit et al., 1991; Labeit and Kolmerer, 1995), and each nebulin super-repeat to associate with a single tropomyosin (Tm)/troponin (Tn) complex (Jin and 


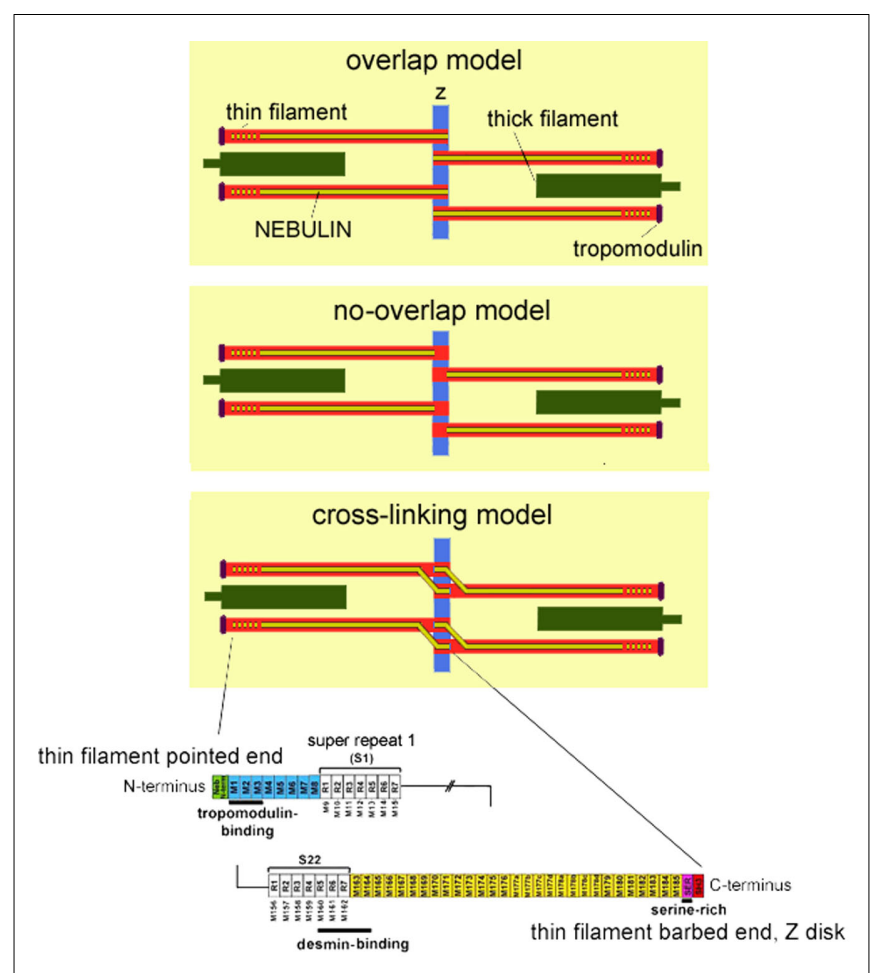

FIGURE 1 | Top:Three schematics of the skeletal muscle sarcomere depicting the three proposed models of nebulin in the Z-disk: the overlap model in which nebulin penetrates the Z-disk creating a $\sim 75 \mathrm{~nm}$ wide nebulin overlap zone in the center of the Z-disk; the no-overlap model in which nebulin penetrates the Z-disk by only about $25 \mathrm{~nm}$, and nebulin filaments from neighboring sarcomeres do not overlap; the cross-linking model in which nebulin fully penetrates the Z-disk and cross-links thin filaments from adjacent sarcomeres. Also note that a single nebulin molecule spans most of the length of the thin filament, but that according to recent studies the thin filament pointed-end is nebulin-free. The length of the nebulin-free thin filament extension is variable (as indicated in the drawing by the dotted nebulin filament) and depends on species and muscle type. Bottom: Schematic of the human nebulin sequence. Nebulin has a highly modular structure, with in the central region (M9-M162) seven modular repeats arranged into 22 super-repeats.

Wang, 1991; McElhinny et al., 2003; Ogut et al., 2003). Nebulin's extreme N-terminal modules M1-M3 (Figure 1) contain a high-affinity binding site for the thin filament pointed-end capping protein tropomodulin (McElhinny et al., 2001). Tropomodulin, in addition to binding nebulin's N-terminus, binds actin and tropomyosin with high-affinity and prevents actin filaments from elongating or shortening at the pointed-end (dos Remedios et al., 2003). Furthermore, earlier studies revealed that the electrophoretic mobility of nebulin from different muscle types correlates with thin filament length (Kruger et al., 1991; Labeit et al., 1991).

Although the findings discussed above were consistent with the hypothesis that nebulin is involved in specifying thin filament length, direct evidence was lacking. More conclusive evidence for a role for nebulin in specifying thin filament length required studies of muscle that lack nebulin. To test the role of nebulin in skeletal muscle in vivo, nebulin $\mathrm{KO}$ mouse models were generated (Bang et al., 2006; Witt et al., 2006). The first work on these models revealed that in nebulin-deficient skeletal muscle the thin filaments are on average shorter, thus supporting a role for nebulin in the in vivo regulation of thin filament length (Bang et al., 2006; Witt et al., 2006). Witt et al. (2006) performed an immunoelectron microscopy study and reported that thin filament lengths in wildtype tibialis cranialis muscle are a constant $1.2 \mu \mathrm{m}$, but in nebulin-deficient muscle are on average $\sim 0.8 \mu \mathrm{m}$, and range from $\sim 0.4$ to $1.2 \mu \mathrm{m}$. That such reduction in thin filament length greatly affects force production was illustrated by Ottenheijm et al. (2009) by plotting force as a function of sarcomere length for both wildtype and nebulin-deficient muscle. In these experiments, skinned muscle fibers were activated by exogenous calcium at various sarcomere lengths and the force response was measured (note that in skinned fiber preparations factors outside of the myofilaments, e.g., calcium handling by the sarcoplasmic reticulum, do not contribute to force production). The force-sarcomere length relation of wildtype muscle is characterized by a force plateau reflecting optimal thick-thin filament overlap, followed by a descending limb at higher sarcomere lengths reflecting the decreased filament overlap. That the descending limb in wildtype muscle starts at a sarcomere length of $\sim 2.6 \mu \mathrm{m}$ and ends at $\sim 4.0 \mu \mathrm{m}$ suggests a thin filament length of $\sim 1.2 \mu \mathrm{m}$, which is in line with the previously mentioned electron microscopy data. In nebulindeficient muscle, the shortened thin filaments reduce thin-thick filament overlap at a given sarcomere length, impairing force production and resulting in a leftward shift of the force-sarcomere length relation (see Figure 2B). Furthermore, when thin filaments are non-uniform in length no optimal thick-thin filament overlap exists, and therefore the force-sarcomere length relation of nebulin-deficient muscle lacks the characteristic plateau. Consistent with these findings on demembranated muscle, studies on intact nebulin-deficient muscle from another nebulin knockout model (Gokhin et al., 2009), in which muscles were activated at various lengths by electrical field stimulation, also revealed a leftward shift of the force-muscle length relation of nebulin-deficient muscle. Thus, the force-length relation of nebulin-deficient muscle is altered in a manner that is consistent with the presence of shorter thin filament lengths.

Work by Bang et al. (2006) on their nebulin KO model, using confocal microscopy on 1-day-old mice, indicated that in the absence of nebulin thin filament lengths are reduced from $\sim 1.15$ to $1.3 \mu \mathrm{m}$ (depending on muscle type) in wildtype muscle to a consistent $\sim 1.0 \mu \mathrm{m}$ in all muscles types. These findings led to the proposal (Littlefield and Fowler, 2008) that a nebulin-independent mechanism specifies uniform thin filament lengths of $\sim 1.0 \mu \mathrm{m}$ in all muscle types, whereas nebulin is responsible for specifying longer thin filament lengths in a muscle-specific manner. An opposite conclusion was drawn by Castillo et al. (2009) who used immuno-fluorescence microscopy on rabbit muscle and concluded that nebulin specifies the minimum thin filament length $(\sim 1.0 \mu \mathrm{m})$ with a nebulin-independent mechanism regulating the final length according to the requirements of a particular muscle. Similar conclusions were drawn recently by Gokhin et al. (2012), showing that human thin filaments have nebulin-free pointed-end extensions that comprise up to $30 \%$ of total thin filament length, 

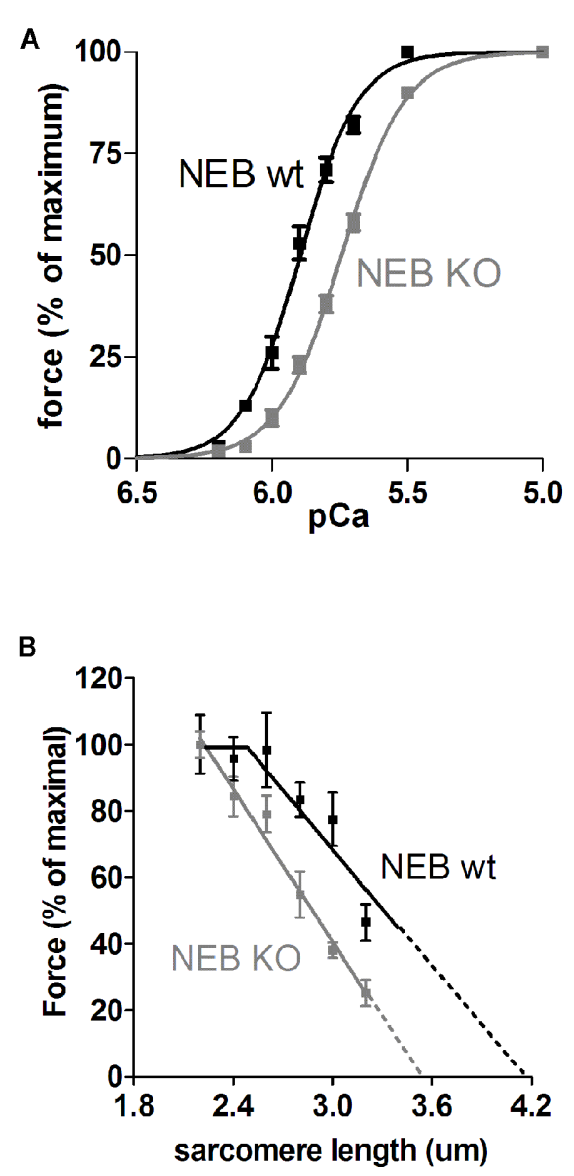

FIGURE 2 | (A) Force-Ca ${ }^{2+}$ characteristics of murine skinned nebulin-deficient muscle fibers from $m$. tibialis cranialis (NEB-KO, left panel), and of skinned muscle fibers from $m$. quadriceps of patients with nebulin-based nemaline myopathy (NEB-NM, right panel). Note that the force generated in response to incubation with incremental increase of $\left[\mathrm{Ca}^{2+}\right]$ is significantly decreased in both NEB-KO and NEB-NM tissue, resulting in a rightward shift of the force-Ca ${ }^{2+}$ relationship (Figures adapted from Chandra et al., 2009;
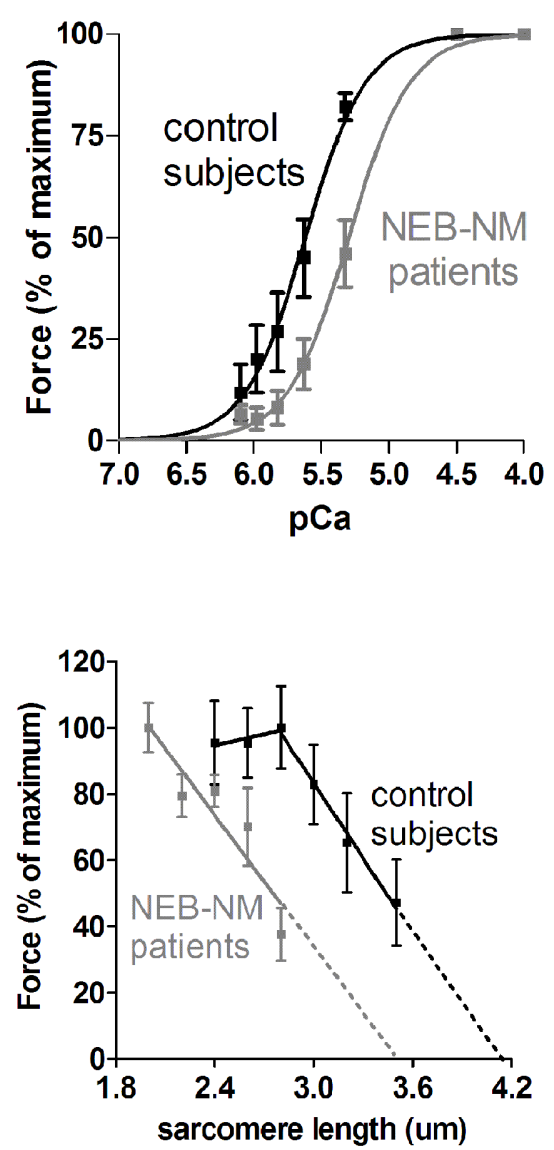

Ottenheijm et al., 2010). (B) Left: the force-sarcomere length relation of murine wt (NEB-wt) fibers has a characteristic force plateau followed by a descending limb. The force-sarcomere length relation of NEB-KO fibers is shifted leftward compared to control fibers, and the force plateau is absent. Right: the force-sarcomere length relation of muscle fibers from NM-NEB patients is shifted to the left and is very similar to that found in NEB-KO fibers (Figures adapted from Ottenheijm et al., 2008). and by Pappas et al. (2010) who showed that when endogenous nebulin is replaced with a mini-nebulin in skeletal myocytes, thin filaments extend beyond the end of mini-nebulin. It is unclear as to what causes this apparent discrepancy between these studies. Detecting differences in thin filament length, especially length gradients, with high precision is challenging and might be hard to accomplish with confocal microscopy, as was done in the two aforementioned studies. In contrast, Witt et al. (2006) used electron microscopy and decorated thin filaments with gold beads (attached to actin monomers with the actin-binding peptide phalloidin). This made it possible to determine thin filament length gradients and showed that thin filaments varied in length and were on average shorter than in wildtype muscle. Thus, this study does not support the conclusion by Bang et al. (2006) that there is a nebulin-independent mechanism that sets a constant thin filament length of $1.0 \mu \mathrm{m}$, but is consistent with Castillo et al. that there is a nebulin-dependent mechanism that sets a minimum thin filament length. To resolve these discrepancies additional studies on a range of mouse muscle types are needed that measure by electron microscopy thin filament length and the location of nebulin's N-terminus. It is clear, however, from the above referenced studies that nebulin does play a critical role in regulating thin filament length: in its absence the average thin filament length is shorter and force is reduced.

\section{NEBULIN AS REGULATOR OF Z-DISK STRUCTURE}

To understand the layout of the C-terminal region of nebulin in the Z-disk, immunoelectron microscopy (IEM) has been used on human soleus muscle and nebulin's C-terminus has been labeled with the nebulin-specific anti-SH3 antibody and the more N-terminal M177-181 domains with anti-neb177-181 (Millevoi et al., 1998). Results showed that the nebulin SH3 domain is located about $25 \mathrm{~nm}$ inside the Z-disk, and the repeats neb176 to neb181 near to the edge of the Z-disk. These results are consistent with two distinct models of the layout of nebulin in the Z-disk (for details, see Millevoi et al., 1998; Figure 1). In the first model, nebulin penetrates the Z-disk by only about $25 \mathrm{~nm}$, and nebulin filaments from neighboring sarcomeres do not overlap (no-overlap model). In the 
second model, nebulin penetrates the Z-disk by about $100 \mathrm{~nm}$ and this creates a $\sim 75 \mathrm{~nm}$ wide nebulin overlap zone in the center of the Z-disk (overlap model). It has been argued that the no-overlap model is more likely to be correct (Millevoi et al., 1998), but definite experimental evidence for either model is lacking. In a more recently proposed model, nebulin fully penetrates the Z-disk and cross-links thin filaments from adjacent sarcomeres (Pappas et al., 2008). A drawback of this model is that it predicts that the SH3 epitope is further from the center of the Z-disk than the M177181 epitope, which is opposite of what has been measured. It also remains to be explained why nebulin leaves one actin filament to which it is anchored and moves to a neighboring filament, and how the thin filament spanning region of nebulin accommodates the myofilament lattice spacing changes that occur during muscle contraction. Clearly further work is needed to map the layout of nebulin in the Z-disk.

Evidence suggests that nebulin's C-terminus regulates Z-disk width. Z-disks of different muscles can vary greatly in width, from less than $100 \mathrm{~nm}$ in fast skeletal muscle to more than $150 \mathrm{~nm}$ in slow skeletal muscle (Tonino et al., 2010). The importance of regulation of Z-disk width is illustrated by muscle from patients with nemaline myopathy, which displays greatly widened Z-disks, including the characteristic nemaline rods (Wallgren-Pettersson et al., 2004). Previously titin has been suggested to play a role in Z-disk assembly (Gautel et al., 1996). The Z-disk region of titin contains a family of differentially expressed repeats, the titin Z-repeats (Gautel et al., 1996). These Z-repeats are a family of $\alpha$-actinin-binding motifs, which are differentially expressed in a tissue- and developmental-stage-specific fashion (Gautel et al., 1996). As previously pointed out, it is unlikely that the differential expression of the titin Z-repeats alone can determine the Z-disk width, because too few isoforms exist to account for the wide range of different Z-disk widths (Millevoi et al., 1998). cDNA sequencing of rabbit nebulin has demonstrated that the Z-disk region of nebulin is differentially expressed (Millevoi et al., 1998). Several different isoforms have been identified that result from the skipping of various combinations of seven Z-disk domains (Millevoi et al., 1998), leading to the suggestion that nebulin is one of several proteins that are important for Z-disk width regulation (Millevoi et al., 1998). Consistent with this notion, the ultrastructural characterization of the Z-disk in NEB-KO mice revealed that average $\mathrm{Z}$-disk width is increased by $40-80 \mathrm{~nm}$ depending on muscle type (Bang et al., 2006; Witt et al., 2006; Tonino et al., 2010). In addition, inclusion-like bodies of mis-assembled Z-disks are detected that resemble the rod bodies that are a hallmark of nemaline myopathy (Bang et al., 2006; Witt et al., 2006). Further support was obtained by a recent study on nebulin in a range of muscle types during postnatal development of the mouse in which transcript studies were performed with a mouse nebulin exon microarray (Buck et al., 2010). During postnatal development of the soleus muscle major changes in splicing were detected in the Z-disk region of nebulin. Three differentially spliced Z-disk exons were upregulated during postnatal development of soleus muscle and this correlated with a significant increase in Z-disk width. The increase in Z-disk width of the soleus muscle might reflect the increasing stress exerted on this muscle due to the rapid increase in body weight during postnatal development.
Together, these findings support a model in which titin and nebulin together specify Z-disk width, with titin constructing the central region of the Z-disk, including the number and positions of $\alpha$-actinin cross-links and nebulin determining the ending of the Z-disk structure and its transition to the I-band, i.e., nebulin functions as a Z-disk terminator. The mechanism by which nebulin terminates the Z-disk might involve interaction between nebulin and Z-disk-localized proteins, such as CapZ. CapZ is a barbed-end actin capping protein that binds near the C-terminus of nebulin (Pappas et al., 2008). In muscle fibers devoid of nebulin (Witt et al., 2006; Pappas et al., 2008), CapZ does not localize properly, allowing the barbed ends of thin filaments to continue to grow beyond the Z-disk resulting in widened Z-disks. Thus, nebulin is involved in regulating Z-disk width. When this feature of nebulin is not present, Z-disks widen, ultimately culminating in the formation of nemaline rods.

\section{NEBULIN MAINTAINS INTERMYOFIBRILLAR CONNECTIVITY}

In addition to regulating Z-disk width and structure, nebulin is suggested to play a role in laterally linking myofibrils at the Z-diks. Longitudinally, sarcomeres are connected by Z-disk lattices that anchor thin filaments and transmit force along the myofibril. In the transverse direction, linkage of myofibrils at the Z-disks allows for lateral force transmission and limits the degree to which adjacent myofibrils translocate relative to each other during active contraction or passive stretch, thereby preventing damage to intermyofibrillar membrane systems, such as T-tubules and the sarcoplasmic reticulum. An important protein involved in linking adjacent Zdisks is the intermediate filament protein desmin, which forms a network of filaments that surrounds myofibrils at the level of the Z-disk (Wang and Ramirez-Mitchell, 1983; Capetanaki et al., 2007). The subunit proteins of desmin filaments are elongated coiled-coils with extensive intermolecular ionic and hydrophobic interactions between individual subunits, giving rise to filaments with high tensile strength as well as plasticity (Costa et al., 2004). That desmin tethers adjacent Z-disks is supported by work on a desmin $\mathrm{KO}$ mouse in which Z-disk misalignment was shown to occur in stretched muscle (Shah et al., 2002). In vitro work, using a yeast two-hybrid approach, suggested that desmin binds to the Cterminal region of nebulin (Bang et al., 2002), which is anchored in the Z-disk, and recently it was shown that nebulin modules M160-170 are involved in this interaction (Conover et al., 2009; Conover and Gregorio, 2011). These findings lead Bang et al. to speculate that this desmin-nebulin interaction links myofibrillar Z-disks to the intermediate filament system, thereby forming a lateral linkage system which maintains adjacent Z-disks in register. This role for nebulin in intermyofibrillar connectivity was tested recently by studies using a nebulin $\mathrm{KO}$ mouse model (Tonino et al., 2010). In these studies it was found that upon stretch, myofibrils devoid of nebulin translocate to a much higher degree than WT muscle, resulting in much larger Z-disk displacement. Although desmin is present in muscle devoid of nebulin, it is reduced in the intermyofibrillar spaces that surround the Z-disks, suggesting that nebulin is required for proper localization of desmin at the Z-disk. Consistent with this, both knockdown of nebulin with siRNA and overexpression of M160-M170 did not interfere with the formation of normal striation patterns but did prevent 
desmin localization at the mature Z-disk. Thus, nebulin is required to laterally link myofibrils at the Z-disk by desmin filaments; in the absence of nebulin myofibrillar connectivity is significantly reduced leading to $\mathrm{Z}$-disk displacement.

\section{NEBULIN AND MUSCLE HYPERTROPHY}

Exciting recent work revealed that nebulin's C-terminal SH3 domain is involved in the induction of muscle hypertrophy. Takano et al. (2010) found that Insulin-like growth factor 1 induces skeletal muscle maturation and hypertrophy by forming a complex of nebulin and N-WASP at the Z-disks of myofibrils by interfering with glycogen synthase kinase- $3 \beta$. Their results convincingly show that N-WASP interacts with nebulin's SH3 domain, and that $\mathrm{N}$-WASP is rapidly recruited to the Z-disk after treatment with insulin-like growth factor 1 , which stimulates myofibrillogenesis and suppresses sarcomere breakdown via the PI3K-Akt pathway. The notion that nebulin is important in maintaining muscle mass is consistent with the previous observation that nebulin-deficient mice display severe muscle fiber atrophy (Bang et al., 2006; Witt et al., 2006) - although thin filament instability caused by nebulindeficiency is likely to be involved as well in the mechanisms underlying muscle atrophy.

\section{NEBULIN REGULATES CONTRACTION}

Recent work shows that nebulin's role is not merely structural, but that nebulin also regulates contraction. Muscle contraction is driven by the cyclic interaction between the myosin-based crossbridges and actin and the level of force a muscle generates is proportional to the force generated per crossbridge and the number of crossbridges in the force generating state. It is generally accepted that this interaction between actin and myosin is regulated through a steric hindrance mechanism in which tropomyosin and troponin control the conversion between interaction permissive and non-permissive states (Gordon et al., 2000). This view needs to be extended as two independent studies (Bang et al., 2009; Chandra et al., 2009) recently identified that nebulin contributes to the regulation of crossbridge cycling kinetics, with one of the two (Chandra et al., 2009) also identifying a role for nebulin in the calcium sensitivity of force generation.

During the crossbridge cycle, unbound non-force generating crossbridges move to an actin-bound force generating state followed by ATP-driven crossbridge release back to the non-force generating state (Huxley and Simmons, 1971; Lymn and Taylor, 1971). Brenner et al. (Brenner, 1988) proposed an analytical framework in which this transition between force and non-force generating crossbridge states can be described by two apparent rate constants; one for crossbridge attachment $\left(f_{\text {app }}\right)$ and one for crossbridge detachment $\left(g_{\text {app }}\right)$. These two rate constants determine the fraction of force generating crossbridges during activation, and a change in one or both will affect this fraction and thus force production. $g_{\text {app }}$ is directly proportional to the ATP consumption rate normalized to tension generation (i.e., tension cost), and can therefore be estimated from the simultaneous determination of ATP consumption rate and tension in activated muscle fibers. Such studies on nebulin-deficient muscle (Chandra et al., 2009) revealed significantly higher tension cost in nebulin-deficient muscle, thus indicating a faster $g_{\text {app }}$ and crossbridge detachment rate when nebulin is absent. Likewise, studies on another nebulin KO mouse model (Bang et al., 2009) reported higher velocity of unloaded shortening in nebulin-deficient muscle, also suggesting that $g_{\text {app }}$ is higher when nebulin is absent. It is also important to highlight that these findings are consistent with results of in vitro motility assays in which nebulin fragments were found to reduce the sliding velocity of F-actin over myosin (Root and Wang, 1994).

In Brenner's framework (Brenner, 1988), the rate constant of force redevelopment $(\mathrm{Ktr})$ is proportional to $f_{\mathrm{app}}+g_{\text {app }}$, and the fraction of force generating crossbridges to $f_{\text {app }} /\left(f_{\text {app }}+g_{\text {app }}\right)$. The rate constant of force redevelopment can be estimated by imposing a rapid release-restretch protocol on an activated fiber, mechanically disengaging all bound crossbridges so that force drops to zero and then measuring force redevelopment. Such experiments revealed that force redevelopment is slower in nebulin-deficient muscle (Bang et al., 2009; Chandra et al., 2009). Thus, the decrease in Ktr of nebulin-deficient muscle, together with the notion that $g_{\text {app }}$ is increased, indicates that $f_{\text {app }}$ must be reduced and that the reduction must be larger than the increase in $g_{\text {app }}$. Combined, this leads to the conclusion that the fraction of force generating crossbridges $\left[f_{\text {app }} /\left(f_{\text {app }}+g_{\text {app }}\right)\right]$ is reduced in nebulindeficient muscle. Furthermore, stiffness measurements indicated that the force per crossbridge was not affected by the absence of nebulin (Bang et al., 2009; Chandra et al., 2009). In summary, recent studies suggest that nebulin increases the rate of crossbridge attachment and reduces the rate of crossbridge detachment, and that as a result the number of force generating crossbridges is increased. Although the mechanism by which nebulin affects crossbridge cycling needs further investigation, previous work (Root and Wang, 1994) has shown that nebulin associates with the actin $\mathrm{N}$-terminus in subdomain 1 , where also the myosin crossbridge binds. Thus, the presence of nebulin at or near the S1 binding site might enhance the binding of crossbridges and slow their detachment. Chandra et al. (2009) estimated that the effect of nebulin on crossbridge kinetics enhances a muscle's force generating capacity by $\sim 50 \%$, and increases the economy of contraction by $\sim 35 \%$. These estimations are in line with findings reported by Bang et al. (2009), and they can largely account for the more pronounced leftward shift of the measured force-sarcomere length relation of nebulin-deficient muscle when compared to the predicted relation based on only thin filament length measurements. Clearly, nebulin is a major factor in determining the level of force and the energetic cost of force production in skeletal muscle. In line with this role of nebulin in the regulation of crossbridge cycling kinetics, recent studies on muscle fibers from patients with nemaline myopathy with severely reduced nebulin protein levels revealed that in addition to altered thin filament length, changes in crossbridge cycling kinetics contribute to the muscle weakness observed in these patients (Ottenheijm et al., 2010).

The studies discussed above were carried out at a maximal activating calcium level. Chandra et al. (2009) also measured active force at a range of calcium levels and the obtained forcepCa relations were markedly shifted to the right in nebulindeficient muscle fibers (see Figure 2A), with a 0.16 unit reduction in $\mathrm{pCa}_{50}$ ( $\mathrm{pCa}$ that gives the half-maximal force level). To 
rule out a possible difference in the troponin complex (isoform composition and posttranslational modification) between wt and KO fibers, these studies were carried out on wildtype and $\mathrm{KO}$ fibers that had been reconstituted by the same recombinant troponin complex. An analysis of expression levels of tropomyosin, myosin heavy chain, myosin light chain did not reveal a significant difference in these proteins between the fibers, suggesting that the absence of nebulin is the most likely explanation for the lower calcium sensitivity of the KO fibers. Interestingly, the studies by Witt et al. (2006) and Bang et al. (2009) found no difference in calcium sensitivity. It is possible that the discrepancy is due to the fact that those studies did not carry out a troponin exchange and that differences in the troponin complex between wt and KO fibers could have negated nebulin's effect on calcium sensitivity. An alternative explanation involves the difference in sarcomere length between the studies. The two studies that did not detect a difference in calcium sensitivity were carried out at long sarcomere lengths $[\sim 2.5 \mu \mathrm{m}$ (Witt et al., 2006) and $\sim 2.6 \mu \mathrm{m}$ (Bang et al., 2009)] whereas the study that did show a difference (Chandra et al., 2009) was performed at $\sim 2.0 \mu \mathrm{m}$. The implication is that nebulin plays a role in the length dependence of activation with a much larger $\Delta \mathrm{pCa}_{50}$ in the nebulin $\mathrm{KO}$ fibers than in wt fibers. It is a well known phenomenon that as sarcomere length increases muscle becomes more calcium sensitive. This length dependence of activation is most prominent in cardiac muscle (and is thought to underlie the Frank-Starling law of the heart) but is much less pronounced in skeletal muscle (Konhilas et al., 2002). The presence of nebulin provides an explanation for why skeletal muscle has less length dependence: the presence of nebulin increases calcium sensitivity at short length. Thus nebulin is an important player in a wide range of skeletal muscle characteristics.

The structure and protein binding properties of nebulin support a role in thin filament activation. Nebulin contains $\sim 200$ domains of $\sim 35$ amino acids that are characterized by the actin-binding sequence SDXXYK; these domains make up seven domain super-repeats characterized by the Tm/Tn binding motif, WLKGIGW (McElhinny et al., 2003). Biochemical studies have shown that a single nebulin module interacts with a single actin monomer and that each nebulin super-repeat interacts with a Tm/Tn complex of the thin filament (Ogut et al., 2003), binding characteristics that support that nebulin follows the helical path of F-actin. It is interesting that similar to Tm, nebulin appears to have different binding sites on $\mathrm{F}$-actin with one site in close proximity to both the strong binding site for myosin and the blocked state of tropomyosin (Lukoyanova et al., 2002). This leads to the intriguing possibility that nebulin acts in concert with Tm and that it promotes the transition of contractile regulatory units ( $\mathrm{Tm} / \mathrm{Tn}$ ) from the non-permissive to the permissive states, thereby increasing myofilament calcium sensitivity.

It is striking that nebulin-deficient skeletal muscle shares the low calcium sensitivity and low maximal active force with cardiac muscle, where stoichiometric levels of nebulin are absent (Bang et al., 2006; Witt et al., 2006). Cardiac muscle contains the nebulin-homolog nebulette (Moncman and Wang, 1995). However, nebulette is much smaller than nebulin $(\sim 100$ vs $\sim 800 \mathrm{kDa}$ for nebulin) and its location is restricted to the Z-disk and the near Z-disk I-band region (Millevoi et al., 1998). Thus, the thin filament in cardiac muscle is largely nebulette-free. Although it can not be ruled out that nebulette induces long-range conformational effects that propagate along the thin filament to affect its function, such effects would have to be first demonstrated in a direct manner, and in absence of such findings we consider it not likely that nebulette is intimately involved in thin filament activation. Cardiac muscle has multiple mechanisms for enhancing thin filament activation, such as, enhanced length-dependent activation, and the presence of multiple cardiac-specific phosphorylation sites in various thin and thick filament based protein sites (Solaro and de Tombe, 2008), which allow cardiac muscle to grade its force response to different loading conditions. Since skeletal muscle lacks the aforementioned features unique to cardiac muscle, nebulin might be essential for tuning thin filament activation for optimal skeletal muscle function.

Changes in calcium homeostasis have also been noted in nebulin-deficient muscle. A striking upregulation of sarcolipin (SLN), an inhibitor of SERCA, occurs in NEB-KO mice (Bang et al., 2006; Gokhin et al., 2009; Ottenheijm et al., 2008). This upregulation might be viewed as an adaptation in NEB-KO mice as an attempt to increase cytosolic calcium levels and counteract reduced myofilament calcium sensitivity. The mechanism by which nebulin-deficiency upregulates SLN requires further future studies, as does the functional role of SLN upregulation, for example by crossing SLN-KO mice and nebulin $\mathrm{KO}$ mice. Taken together, the above discussed studies clearly indicate that nebulin is not merely involved in regulation sarcomere structure, but also acts as a regulator of muscle contraction.

\section{NEBULIN AND NEMALINE MYOPATHY}

Dysfunctions of the skeletal muscle thin filament have emerged as important causes of skeletal myopathies of which nemaline myopathy is the most common disease with a previously estimated prevalence of $0.002 \%$ (Sanoudou and Beggs, 2001). However, because of its heterogeneous nature and often milder phenotypes, the actual frequency of nemaline myopathy in older patients may be considerably higher. Genetically, NM is heterogeneous and so far seven genes have been identified as NM-causing, namely alphatropomyosin-3 and beta-tropomyosin (TPM3 and TPM2), NEB, actin alpha 1 (ACTA1), troponin T type 1 (TNNT1), cofilin-2, and kbtbd13 (Laing et al., 1992, 1995; Nowak et al., 1999; Pelin et al., 1999; Ryan et al., 2001; Wallgren-Pettersson et al., 2002, 2004; Agrawal et al., 2007; Sambuughin et al., 2010). An unbiased linkage analysis of $45 \mathrm{NM}$ families derived from 10 different countries implicated nebulin mutations as disease causing in 41 of these families. The typical form of NM presents with early onset in infancy, has a non-progressive or slowly progressive course and is caused by mutations in the nebulin gene. These mutations are often missense mutations, but in addition a 2502-bp deletion causing a 33-residue in frame deletion of exon 55 was identified as a prominent recessive disease causing allele initially in the Ashkenazi Jewish population, and more recently also in non-Jewish patients (Anderson et al., 2004; Lehtokari et al., 2006, 2009). 
Recent studies on muscle fibers from patients with nemaline myopathy due to the deletion of nebulin exon 55 showed that these patients have severely reduced nebulin protein levels and that they show remarkable phenotypic similarities to fibers from nebulin $\mathrm{KO}$ mice, i.e., shorter and non-uniform thin filament lengths (Figure 2B) and significantly impaired force generating capacity (Ottenheijm et al., 2009). Thus, loss of thin filament length regulation appears to be an important contributor to muscle weakness in patients with nemaline myopathy. In addition to altered thin filament length, changes in crossbridge cycling kinetics and reduced calcium sensitivity of force production contribute to the muscle weakness observed in these patients (see Figure 2A; Ottenheijm et al., 2010), in line with the role of nebulin in these processes (Chandra et al., 2009; Ottenheijm and Granzier, 2010). Thus, nebulin's role in thin filament length regulation and contraction deduced from work on NEB-KO mice provides a mechanism for the first time to explain severe muscle weakness in patients with nemaline myopathy. Interestingly, recent work form our lab demonstrates that patients with nemaline myopathy due to mutations in the tropomyosin gene (TPM3) display a contractile phenotype that is distinct from that of patients with NEB-based myopathy (Ottenheijm et al., 2011). Whereas both show severe myofilament-based muscle weakness, the contractile dysfunction in TPM3-based myopathy can be largely explained by changes in crossbridge cycling kinetics, whereas dysregulation of

\section{REFERENCES}

Agrawal, P. B., Greenleaf, R. S., Tomczak, K. K., Lehtokari, V. L., WallgrenPettersson, C., Wallefeld, W., Laing, N. G., Darras, B. T., Maciver, S. K., Dormitzer, P. R., and Beggs, A. H. (2007). Nemaline myopathy with minicores caused by mutation of the CFL2 gene encoding the skeletal muscle actin-binding protein, cofilin-2. Am. J. Hum. Genet. 80, 162-167.

Anderson, S. L., Ekstein, J., Donnelly, M. C., Keefe, E. M., Toto, N. R., LeVoci, L. A., and Rubin, B. Y. (2004). Nemaline myopathy in the Ashkenazi Jewish population is caused by a deletion in the nebulin gene. Hum. Genet. 115, 185-190.

Bang, M. L., Caremani, M., Brunello, E., Littlefield, R., Lieber, R. L., Chen, J., Lombardi, V., and Linari, M. (2009). Nebulin plays a direct role in promoting strong actin-myosin interactions. FASEB J. 23, 4117-4125.

Bang, M. L., Gregorio, C., and Labeit, S. (2002). Molecular dissection of the interaction of desmin with the Cterminal region of nebulin. J. Struct. Biol. 137, 119-127.

Bang, M. L., Li, X., Littlefield, R., Bremner, S., Thor, A., Knowlton, K. U., Lieber, R. L., and Chen, J. (2006). Nebulin-deficient mice exhibit shorter thin filament lengths and reduced contractile function in

thin filament length and altered crossbridge cycling kinetics play a prominent role in NEB-based myopathy (Ottenheijm et al., 2009). Furthermore, the loss of force generating capacity in TPM3-based myopathy appears to be partly compensated by enhanced calcium sensitivity of force generation, whereas decreased calcium sensitivity of force generation further depresses the capacity for force production in NEB-based myopathy (Ottenheijm et al., 2010). Whereas these findings support the existence of distinct genotypephenotype correlations in NM, it is yet unclear whether they extrapolate to other patients with mutations in NEB or TPM3, and how they compare to the phenotype of NM patients with mutations in ACTA1, TPM2, TNNT1, CFL2, and KBTBD13. Understanding the genotype-phenotype correlations is important, as it allows the development of genotype-targeted treatment strategies. For example, analogous to current clinical studies that address whether contractility during heart failure can be augmented by actomyosin activating small molecules (Malik et al., 2011), a rationale for similar approaches in $N E B$-based nemaline myopathy now exists.

\section{ACKNOWLEDGMENTS}

This work was funded by the seventh Framework Program of the European Union (project "NEMMYOP") to Coen A. C. Ottenheijm and Siegfried Labeit, and by National Institutes of Health grant RO1 AR053897 to Henk Granzier.

filament activation: a novel mechanism for increasing tension and reducing tension cost. J. Biol. Chem. 284, 30889-30896.

Conover, G. M., and Gregorio, C. (2011). The desmin coil 1B mutation K190A impairs nebulin Zdisc assembly and destabilizes actin thin filaments. J. Cell. Sci. 124, 3463-3476.

Conover, G. M., Henderson, S. N., and Gregorio, C. C. (2009). A myopathylinked desmin mutation perturbs striated muscle actin filament architecture. Mol. Biol. Cell 20, 834-845.

Costa, M. L., Escaleira, R., Cataldo, A., Oliveira, F., and Mermelstein, C. S. (2004). Desmin: molecular interactions and putative functions of the muscle intermediate filament protein. Braz. J. Med. Biol. Res. 37, 1819-1830.

dos Remedios, C. G., Chhabra, D., Kekic, M., Dedova, I. V., Tsubakihara, M., Berry, D. A., and Nosworthy, N. J. (2003). Actin binding proteins: regulation of cytoskeletal microfilaments. Physiol. Rev. 83, 433-473.

Gautel, M., Goulding, D., Bullard, B., Weber, K., and Furst, D. O. (1996). The central Z-disk region of titin is assembled from a novel repeat in variable copy numbers. J. Cell. Sci. 109(Pt 11), 2747-2754.

Gokhin, D. S., Bang, M. L., Zhang, J., Chen, J., and Lieber, R. L. (2009).
Reduced thin filament length in nebulin-knockout skeletal muscle alters isometric contractile properties. Am. J. Physiol. Cell Physiol. 296, C1123-C1132.

Gokhin, D. S., Kim, N. E., Lewis, S. A., Hoenecke, H. R., D'Lima, D. D., and Fowler, V. M. (2012). Thin filament length correlates with fiber type in human skeletal muscle. Am. J. Physiol. Cell Physiol. 302, C555-C565.

Gordon, A. M., Homsher, E., and Regnier, M. (2000). Regulation of contraction in striated muscle. Physiol. Rev. 80, 853-924.

Granzier, H. L., Akster, H. A., and Ter Keurs, H. E. (1991). Effect of thin filament length on the forcesarcomere length relation of skeletal muscle. Am. J. Physiol. 260, C1060-C1070.

Huxley, A. F., and Simmons, R. M. (1971). Proposed mechanism of force generation in striated muscle. Nature 233, 533-538.

Jin, J. P., and Wang, K. (1991). Cloning, expression, and protein interaction of human nebulin fragments composed of varying numbers of sequence modules. J. Biol. Chem. 266, 21215-21223.

Konhilas, J. P., Irving, T. C., and de Tombe, P. P. (2002). Lengthdependent activation in three striated muscle types of the rat. J. Physiol. (Lond.) 544, 225-236. 
Kruger, M., Wright, J., and Wang, K. (1991). Nebulin as a length regulator of thin filaments of vertebrate skeletal muscles: correlation of thin filament length, nebulin size, and epitope profile. J. Cell Biol. 115, 97-107.

Labeit, S., Gibson, T., Lakey, A., Leonard, K., Zeviani, M., Knight, P., Wardale, J., and Trinick, J. (1991). Evidence that nebulin is a protein-ruler in muscle thin filaments. FEBS Lett. 282, 313-316.

Labeit, S., and Kolmerer, B. (1995). The complete primary structure of human nebulin and its correlation to muscle structure. J. Mol. Biol. 248, 308-315.

Laing, N. G., Majda, B. T., Akkari, P. A., Layton, M. G., Mulley, J. C., Phillips, H., Haan, E. A., White, S. J., Beggs, A. H., and Kunkel, L. M. (1992). Assignment of a gene (NEMI) for autosomal dominant nemaline myopathy to chromosome I. Am. J. Hum. Genet. 50, 576-583.

Laing, N. G., Wilton, S. D., Akkari, P. A., Dorosz, S., Boundy, K., Kneebone, C., Blumbergs, P., White, S., Watkins, H., and Love, D. R. (1995). A mutation in the alpha tropomyosin gene TPM3 associated with autosomal dominant nemaline myopathy NEM1. Nat. Genet. 10, 249.

Lehtokari, V. L., Greenleaf, R. S., Dechene, E. T., Kellinsalmi, M., Pelin, K., Laing, N. G., Beggs, A. H., and Wallgren-Pettersson, C. (2009). The exon 55 deletion in the nebulin gene - one single founder mutation with world-wide occurrence. Neuromuscul. Disord. 19, 179-181.

Lehtokari, V. L., Pelin, K., Sandbacka, M., Ranta, S., Donner, K., Muntoni, F., Sewry, C., Angelini, C., Bushby, K., Van den, B. P., Iannaccone, S., Laing, N. G., and WallgrenPettersson, C. (2006). Identification of 45 novel mutations in the nebulin gene associated with autosomal recessive nemaline myopathy. Hum. Mutat. 27, 946-956.

Littlefield, R. S., and Fowler, V. M. (2008). Thin filament length regulation in striated muscle sarcomeres: pointed-end dynamics go beyond a nebulin ruler. Semin. Cell Dev. Biol. 19, 511-519.

Lukoyanova, N., VanLoock, M. S., Orlova, A., Galkin, V. E., Wang, K., and Egelman, E. H. (2002). Each actin subunit has three nebulin binding sites: implications for steric blocking. Curr. Biol. 12, 383-388.

Lymn, R. W., and Taylor, E. W. (1971). Mechanism of adenosine triphosphate hydrolysis by actomyosin. Biochemistry 10, 4617-4624.

Malik, F. I., Hartman, J. J., Elias, K. A., Morgan, B., Rodriguez, H., Brejc, K., Anderson, R. L., Sueoka, S. H., Lee, K. H., Finer, J. T., Sakowicz, R., Baliga, R., Cox, D. R., Garard, M., Godinez, G., Kawas, R., Kraynack, E., Lenzi, D., Lu, P. P., Muci, A., Niu, C., Qian, X., Pierce, D. W., Pokrovskii, M., Suehiro, I., Sylvester, R., Tochimoto, T., Valdez, C., Wang, W., Katori, T., Kass, D. A., Shen, Y. T., Vatner, S. F., and Morgans, D. J. (2011). Cardiac myosin activation: a potential therapeutic approach for systolic heart failure. Science 331, 1439-1443.

McElhinny, A. S., Kazmierski, S. T., Labeit, S., and Gregorio, C. C. (2003). Nebulin: the nebulous, multifunctional giant of striated muscle. Trends Cardiovasc. Med. 13, 195-201.

McElhinny, A. S., Kolmerer, B., Fowler, V. M., Labeit, S., and Gregorio, C. C. (2001). The N-terminal end of nebulin interacts with tropomodulin at the pointed ends of the thin filaments. J. Biol. Chem. 276, 583-592.

Millevoi, S., Trombitas, K., Kolmerer, B., Kostin, S., Schaper, J., Pelin, K., Granzier, H., and Labeit, S. (1998). Characterization of nebulette and nebulin and emerging concepts of their roles for vertebrate Z-discs. J. Mol. Biol. 282, 111-123.

Moncman, C. L., and Wang, K. (1995). Nebulette: a $107 \mathrm{kD}$ nebulin-like protein in cardiac muscle. Cell Motil. Cytoskeleton 32, 205-225.

Nowak, K. J., Wattanasirichaigoon, D., Goebel, H. H., Wilce, M., Pelin, K., Donner, K., Jacob, R. L., Hubner, C., Oexle, K., Anderson, J. R., Verity, C. M., North, K., Iannaccone, S., Muller, C. R., Nurnberg, P., Muntoni, F., Sewry, C., Hughes, I., Sutphen, R., Lacson, A. G., Swoboda, K. J., Vigneron, J., WallgrenPettersson, C., Beggs, A. H., and Laing, N. G. (1999). Mutations in the skeletal muscle alpha-actin gene in patients with actin myopathy and nemaline myopathy. Nat. Genet. 23, 208-212.

Ogut, O., Hossain, M. M., and Jin, J. P. (2003). Interactions between nebulin-like motifs and thin filament regulatory proteins. J. Biol. Chem. 278, 3089-3097.

Ottenheijm, C. A., Fong, C., Vangheluwe, P., Wuytack, F., Babu, G. J., Periasamy, M., Witt, C. C., Labeit, S., and Granzier, H.
(2008). Sarcoplasmic reticulum calcium uptake and speed of relaxation are depressed in nebulinfree skeletal muscle. FASEB J. 22, 2912-2919.

Ottenheijm, C. A., and Granzier, H. (2010). Lifting the nebula: novel insights into skeletal muscle contractility. Physiology (Bethesda) 25, 304-310.

Ottenheijm, C. A., Hooijman, P., Dechene, E. T., Stienen, G. J., Beggs, A. H., and Granzier, $\mathrm{H}$. (2010). Altered myofilament function depresses force generation in patients with nebulin-based nemaline myopathy (NEM2). J. Struct. Biol. 170, 334-343.

Ottenheijm, C. A., Lawlor, M. W., Stienen, G. J., Granzier, H., and Beggs, A. H. (2011). Changes in cross-bridge cycling underlie muscle weakness in patients with tropomyosin 3-based myopathy. Hum. Mol. Genet. 20, 2015-2025.

Ottenheijm, C. A., Witt, C. C., Stienen, G. J., Labeit, S., Beggs, A. H., and Granzier, H. (2009). Thin filament length dysregulation contributes to muscle weakness in nemaline myopathy patients with nebulin deficiency. Hum. Mol. Genet. 18, 2359-2369.

Pappas, C. T., Bhattacharya, N., Cooper, J. A., and Gregorio, C. C. (2008). Nebulin interacts with CapZ and regulates thin filament architecture within the Z-disc. Mol. Biol. Cell 19, 1837-1847.

Pappas, C. T., Krieg, P. A., and Gregorio, C. C. (2010). Nebulin regulates actin filament lengths by a stabilization mechanism. J. Cell Biol. 189, 859-870.

Pelin, K., Hilpela, P., Donner, K., Sewry, C., Akkari, P. A., Wilton, S. D., Wattanasirichaigoon, D., Bang, M. L., Centner, T., Hanefeld, F., Odent, S., Fardeau, M., Urtizberea, J. A., Muntoni, F., Dubowitz, V., Beggs, A. H., Laing, N. G., Labeit, S., de la, C. A., and Wallgren-Pettersson, C. (1999). Mutations in the nebulin gene associated with autosomal recessive nemaline myopathy. Proc. Natl. Acad. Sci. U.S.A. 96, 2305-2310.

Pollard, T. D., and Borisy, G. G. (2003). Cellular motility driven by assembly and disassembly of actin filaments. Cell 112, 453-465.

Root, D. D., and Wang, K. (1994). Calmodulin-sensitive interaction of human nebulin fragments with actin and myosin. Biochemistry 33, 12581-12591.

Ryan, M. M., Schnell, C., Strickland, C. D., Shield, L. K., Morgan, B.,
Iannaccone, S., Laing, N. G., Beggs, A. H., and North, K. (2001). Nemaline myopathy: a clinical study of 143 cases. Ann. Neurol. 50, 312-320.

Sambuughin, N., Yau, K. S., Olive, M., Duff, R. M., Bayarsaikhan, M. Lu, S., Gonzalez-Mera, L., Sivadorai, P., Nowak, K. J., Ravenscroft, G., Mastaglia, F. L., North, K. N., Ilkovski, B., Kremer, H., Lammens, M., van Engelen, B. G., Fabian, V., Lamont, P., Davis, M. R., Laing, N. G., and Goldfarb, L. G. (2010). Dominant mutations in KBTBD13, a member of the BTB/Kelch family, cause nemaline myopathy with cores. Am. J. Hum. Genet. 87, 842-847.

Sanoudou, D., and Beggs, A. H. (2001). Clinical and genetic heterogeneity in nemaline myopathy a disease of skeletal muscle thin filaments. Trends Mol. Med. 7, 362-368.

Shah, S. B., Su, F. C., Jordan, K., Milner, D. J., Friden, J., Capetanaki, Y., and Lieber, R. L. (2002). Evidence for increased myofibrillar mobility in desmin-null mouse skeletal muscle. J. Exp. Biol. 205, 321-325.

Solaro, R. J., and de Tombe, P. P. (2008). Review focus series: sarcomeric proteins as key elements in integrated control of cardiac function. Cardiovasc. Res. 77, 616-618.

Takano, K., Watanabe-Takano, H., Suetsugu, S., Kurita, S., Tsujita, K., Kimura, S., Karatsu, T., Takenawa, T., and Endo, T. (2010). Nebulin and N-WASP cooperate to cause IGF-1-induced sarcomeric actin filament formation. Science 330, 1536-1540.

Tonino, P., Pappas, C. T., Hudson, B. D., Labeit, S., Gregorio, C. C., and Granzier, H. (2010). Reduced myofibrillar connectivity and increased Z-disk width in nebulin-deficient skeletal muscle. $J$. Cell Sci. 123, 384-391.

Wallgren-Pettersson, C., Donner, K., Sewry, C., Bijlsma, E., Lammens, M., Bushby, K., Giovannucci Uzielli, M. L., Lapi, E., Odent, S., Akcoren, Z., Topaloglu, H., and Pelin, K. (2002). Mutations in the nebulin gene can cause severe congenital nemaline myopathy. Neuromuscul. Disord. 12, 674-679.

Wallgren-Pettersson, C., Pelin, K., Nowak, K. J., Muntoni, F., Romero, N. B., Goebel, H. H., North, K. N., Beggs, A. H., and Laing, N. G. (2004). Genotype-phenotype correlations in nemaline myopathy caused by mutations in the genes for nebulin and skeletal muscle 
alpha-actin. Neuromuscul. Disord. 14, 461-470.

Wang, K., and Ramirez-Mitchell, R. (1983). A network of transverse and longitudinal intermediate filaments is associated with sarcomeres of adult vertebrate skeletal muscle. J. Cell Biol. 96, 562-570.

Wang, K., and Wright, J. (1988). Architecture of the sarcomere matrix of skeletal muscle: immunoelectron microscopic evidence that suggests a set of parallel inextensible nebulin filaments anchored at the $\mathrm{Z}$ line. J. Cell Biol. 107, 2199-2212.

Witt, C. C., Burkart, C., Labeit, D., McNabb, M., Wu, Y., Granzier, H., and Labeit, S. (2006). Nebulin regulates thin filament length, contractility, and Z-disk structure in vivo. EMBO J. 25, 3843-3855.

Conflict of Interest Statement: The authors declare that the research was conducted in the absence of any commercial or financial relationships that could be construed as a potential conflict of interest.

Received: 10 January 2012; accepted: 09 February 2012; published online: 27 February 2012.

Citation: Ottenheijm CAC, Granzier $H$ and Labeit $S$ (2012) The sarcomeric protein nebulin: another multifunctional giant in charge of muscle strength optimization. Front.
Physio. 3:37. doi: 10.3389/fphys.2012. 00037

This article was submitted to Frontiers in Striated Muscle Physiology, a specialty of Frontiers in Physiology.

Copyright (C) 2012 Ottenheijm, Granzier and Labeit. This is an open-access article distributed under the terms of the Creative Commons Attribution Non Commercial License, which permits noncommercial use, distribution, and reproduction in other forums, provided the original authors and source are credited. 